\title{
Freqüência alimentar e crescimento de alevinos de jundiá, Rhamdia quelen
}

\author{
Feeding frequency and growth of silver catfish, Rhamdia quelen, fingerlings
}

Paulo César Falanghe Carneiro ${ }^{1}$ Jorge Daniel Mikos ${ }^{2}$

\section{RESUMO}

O jundiá, Rhamdia quelen, é uma espécie de peixe nativa ainda pouco conhecida cientificamente, mas que tem atraído a atenção de produtores e pesquisadores devido a várias características favoráveis à sua inclusão na lista de peixes criados comercialmente no país. A freqüência alimentar é um fator importante dentro do manejo em piscicultura visando melhorar a eficiência produtiva e reduzir os custos de produção. $O$ presente trabalho testou quatro regimes de arraçoamento $(1,2,3$ e 4 vezes ao dia) em intervalos regulares. Alevinos de jundiá $(1,88 \mathrm{~g})$ foram estocados em 12 tanques de $4 \mathrm{~m}^{2}$ por 65 dias. A temperatura da água variou de 23 a $27^{\circ} \mathrm{C}$, durante o período experimental, e os peixes de todos os tratamentos apresentaram índices zootécnicos de desempenho (ganho de peso, conversão alimentar $e$ crescimento especifico) semelhantes. Os peixes alimentados apenas uma vez ao dia tiveram crescimento semelhante àqueles alimentados duas a quatro vezes ao dia. Estes resultados contribuem para a otimização dos recursos necessários à produção comercial do jundiá em cativeiro.

Palavras-chave: Rhamdia quelen, jundiá, regime alimentar.

\section{ABSTRACT}

Silver catfish Rhamdia quelen is an important species commonly found in many Brazilian rivers and other regions of South America. Studies carried out in the last years with this species have pointed its great potential for aquaculture in Brazil, mainly in the Southern States. Feeding frequency is a very important issue for fish producers due to its direct relation to production costs. The present study tested four different feeding frequencies (1, 2, 3, and 4 times a day). Forty jundiá fingerlings (1.88g) were stocked in each of the twelve 4- $\mathrm{m}^{2}$ tanks (concrete wall and earth bottom) and raised for 65 days. Water temperature ranged from 23 to $27^{\circ} \mathrm{C}$ during the experimental period and fish from all treatments showed the same growth pattern. Body weight and length, feed conversion rate, and specific growth rate were not significantly different among the treatments. Silver catfish fed only once a day grew similarly to those fed two to four times a day. These findings could optimize farm labor and increase profit in silver catfish production.

Key words: Rhamdia quelen, catfish, feeding regime.

\section{INTRODUÇÃO}

O jundiá é um peixe que apresenta grande aceitação pelo mercado consumidor devido à sua carne saborosa e ausência de espinhos intramusculares. Os adultos desta espécie são onívoros no ambiente natural, tendo preferência por peixes, crustáceos, insetos, restos vegetais e detritos orgânicos. Os organismos encontrados no conteúdo gastrintestinal do jundiá não são restritos ao habitat bentônico, indicando que essa espécie é generalista com relação à escolha do alimento (GOMES et al., 2000). Esta característica contribui para a adaptação deste peixe ao alimento artificial, facilitando sobremaneira a sua domesticação e condicionamento às condições de cultivo.

SILFVERGRIP (1996) observou que a sistemática do gênero Rhamdia é confusa desde que foi descrita, sendo este gênero formado por apenas 11 espécies e não pelas 100 anteriormente descritas. O mesmo autor verificou que apenas o jundiá, Rhamdia quelen, apresenta 49 sinonímias, reforçando a

\footnotetext{
${ }^{1}$ Engenheiro Agrônomo, Professor Adjunto do Curso de Zootecnia, Centro de Ciências Agrárias e Ambientais (CCAA), Pontifícia Universidade Católica do Paraná (PUCPR).

${ }^{2}$ Acadêmico de Agronomia, Bolsista do Programa Institucional de Bolsas de Iniciação Científica (PIBIC), Conselho Nacional de Desenvolvimento Científico e Tecnológico (CNPq), CCAA, PUCPR. Autor para correspondência: PUCPR, CCAA, BR 376, km 14, CP 129, 83010-050, São José dos Pinhais, PR. E-mail: paulo.carneiro@pucpr.br.
} 
necessidade de mais estudos sobre esta espécie. Esta espécie de peixe é ainda pouco conhecida cientificamente, mas tem atraído a atenção de produtores e pesquisadores devido a várias características favoráveis a sua inclusão na lista de peixes criados comercialmente no país, principalmente na região sul do Brasil (GOMES et al., 2000; CARNEIRO et al., 2002, 2003).

A freqüência do fornecimento do alimento é um fator importante dentro do manejo alimentar por estimular o peixe a procurar pelo alimento em momentos pré-determinados, podendo contribuir para a redução na conversão alimentar, incrementar o ganho de peso, além de possibilitar maior oportunidade de observação do estado de saúde dos peixes. O conhecimento do número mais adequado de arraçoamento contribui ainda para a redução do desperdício de alimento, garantindo a qualidade da água e reduzindo os custos de produção.

Um dos fatores que determinam a freqüência alimentar dos peixes é o estágio de desenvolvimento dos animais, sendo que peixes jovens (pós-larvas e alevinos) apresentam maior atividade metabólica e necessitam de maior freqüência no fornecimento do alimento em relação aos animais adultos (MURAI \& ANDREWS, 1976; FOLKVORD \& OTTERA, 1993). Espécies de peixes onívoros com estômago pequeno, como a tilápia Oreochromis niloticus, procuram o alimento mais freqüentemente por apresentarem limitação na capacidade de armazenamento de alimento. Já as espécies carnívoras e algumas onívoras possuem estômago grande e podem ingerir grande quantidade de alimentos num único momento, mantendo-se saciados por um longo período (TUCKER \& ROBINSON, 1991).

O objetivo do presente trabalho foi avaliar o desempenho produtivo do jundiá sob o efeito de diferentes regimes de fornecimento de ração (freqüência alimentar).

\section{MATERIAL E MÉTODOS}

O presente experimento foi conduzido entre 19 de março e 20 de maio de 2002 no Setor de Piscicultura da PUCPR (São José dos Pinhais - PR) em três tanques de $16 \mathrm{~m}^{2}$ expostos ao ar livre e protegidos contra predadores aéreos por telas de nylon suspensas a $30 \mathrm{~cm}$ da superfície da água. Estes tanques foram revestidos lateralmente de alvenaria e receberam uma mistura de terra e areia no fundo para aproximar suas condições ambientais àquelas normalmente encontradas num viveiro de piscicultura. Cada tanque foi dividido com tela plástica de $5 \mathrm{~mm}$, formando quatro parcelas com $4 \mathrm{~m}^{2}$ cada. O suprimento contínuo de ar foi feito por pedras porosas ligadas a um compressor de ar de 5cv. O abastecimento de água, feito através de um poço semi-artesiano, era responsável apenas pela reposição do volume perdido pela evaporação e infiltração, não havendo, portanto renovação e sim manutenção do volume constante.

Os tratamentos foram constituídos de quatro regimes de arraçoamento: 1, 2, 3 e 4 vezes ao dia (com 3 repetições) em intervalos regulares (9h; 11h; $13 \mathrm{~h} 15 \mathrm{~h})$. Cada parcela recebeu 40 alevinos (comprimento médio 6cm e peso médio 1,88g) que foram submetidos aos tratamentos por 65 dias. A ração foi fornecida “ad libitum” (ração flutuante 2-4mm Nutripeixe TR 36 Purina Agribands do Brasil Ltda) após triturada e peneirada em malha $2 \mathrm{~mm}$. O crescimento dos peixes foi monitorado 23, 45 e 65 dias após o início do experimento, sendo calculados a conversão alimentar aparente [quantidade de ração fornecida (g) / ganho de peso (g)] e o crescimento específico [100 x (ln peso final - ln peso inicial)/tempo (dias) - \%/dia]. Durante as primeiras biometrias foram registrados o peso e comprimento total de 15-30 peixes por parcela. Ao final do período experimental, todos os peixes foram medidos e pesados individualmente. O monitoramento da qualidade da água foi realizado diariamente às $9 \mathrm{~h}$ através da análise do $\mathrm{pH}$, oxigênio dissolvido e temperatura.

Análise estatística

O delineamento experimental foi em blocos inteiramente casualizados, sendo que cada um dos três tanques recebeu uma repetição de cada um dos tratamentos. Os dados obtidos no experimento foram submetidos à análise de variância pelo teste $\mathrm{F}$, sendo os resultados apresentados como médias acompanhadas do desvio padrão. O nível de significância considerado foi de $5 \%(\mathrm{P}<0,05)$. As análises foram feitas com auxílio do programa estatístico Statistica ${ }^{\circledR} 6.0$ (StatSoft, Inc.).

\section{RESULTADOS E DISCUSSÃO}

Durante o período experimental, a água de todos os tanques apresentou transparência 48,8 \pm 7,0cm, pH 8,0 \pm 0,5, oxigênio dissolvido 9,8 $\pm 2,2 \mathrm{mg}$ $\mathrm{L}^{-1}$ e temperatura $25,2 \pm 1,3^{\circ} \mathrm{C}$. Estes valores são considerados normais para a maioria dos viveiros com espécies tropicais, não interferindo, portanto, nos resultados obtidos no experimento.

As médias de ganho de peso (Figura 1), conversão alimentar (Figura 2) e crescimento específico 


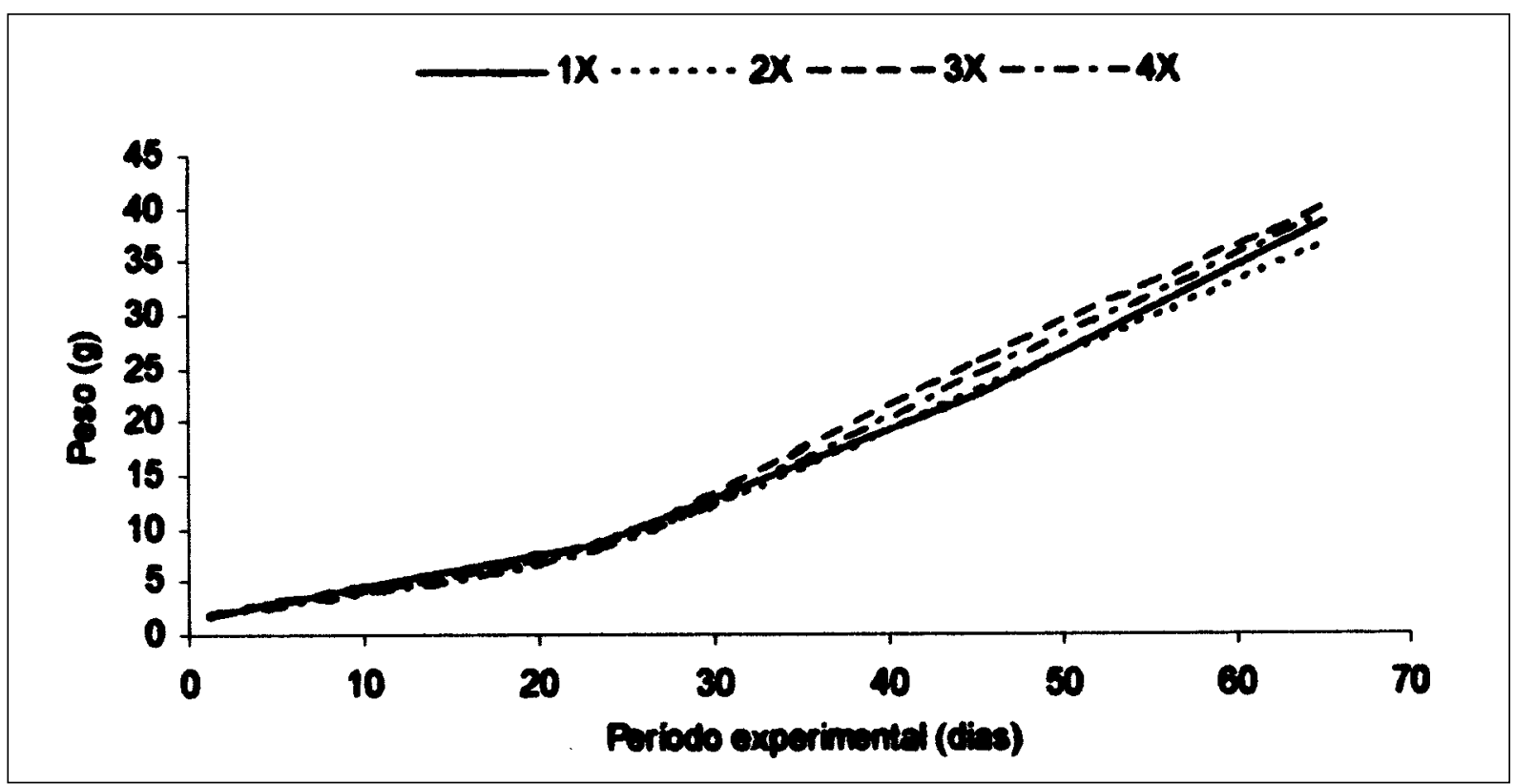

Figura 1 - Crescimento de alevinos de jundiá submetidos a diferentes regimes de fornecimento de ração (1, 2, 3, e 4 vezes ao dia) durante 65 dias.

(Figura 3) permaneceram semelhantes entre os tratamentos ( $\mathrm{p}>0,05)$. O efeito da freqüência alimentar no crescimento e consumo do peixe pode variar de espécie para espécie. THOMASSEN \& FJAERA (1996) observaram em salmão do Atlântico, Salmo salar, aumento no consumo de alimento e melhor crescimento associado ao aumento na freqüência alimentar. RUOHONEN et al. (1998) forneceram dois tipos de dietas (seca e úmida) para truta, Oncorhynchus mykiss, 1,2 e 4 vezes ao dia e também observaram aumento no crescimento dos peixes associado ao aumento na freqüência alimentar. Outro experimento semelhante

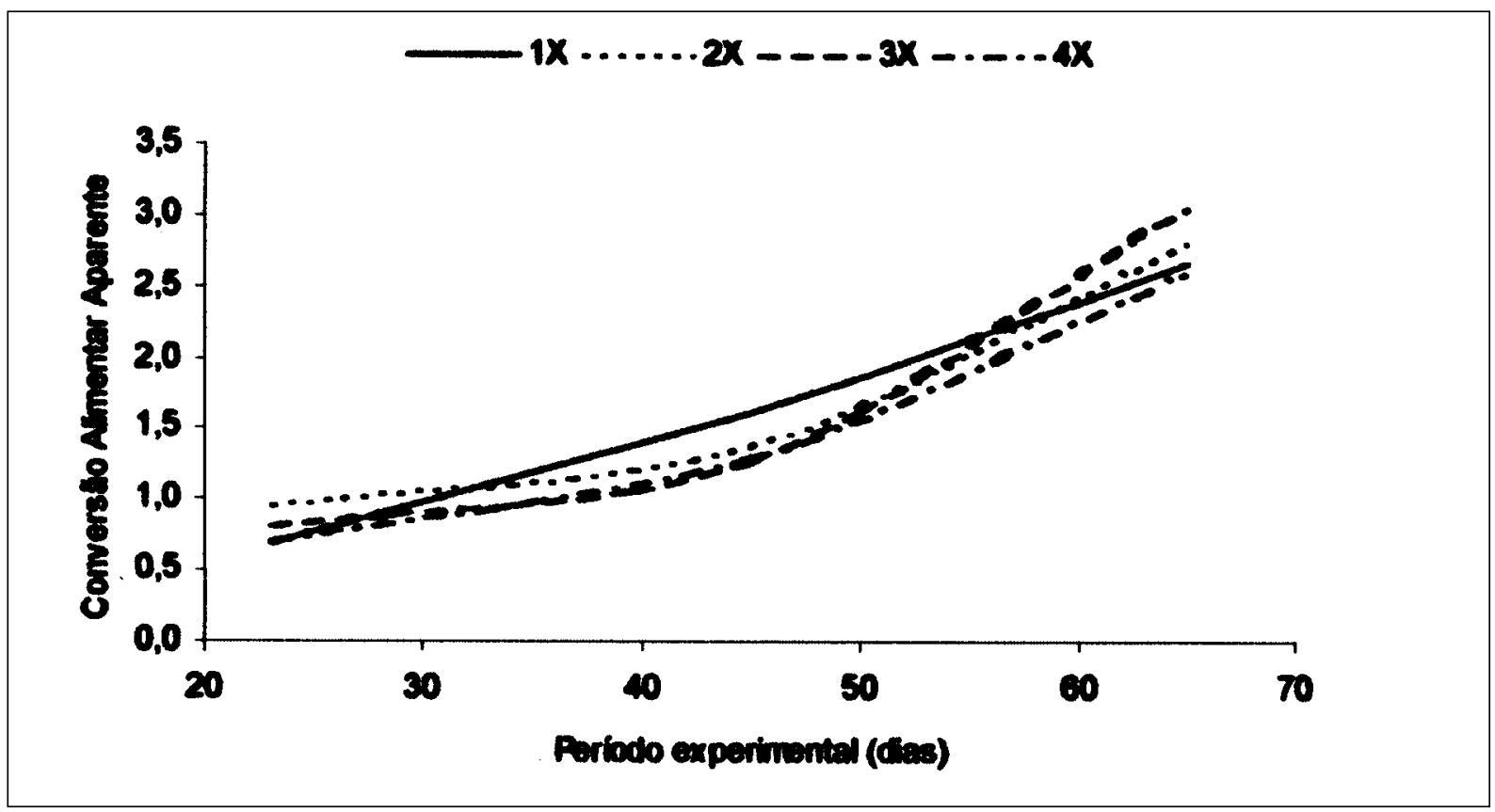

Figura 2 - Conversão alimentar aparente de alevinos de jundiá submetidos a diferentes regimes de fornecimento de ração (1, 2, 3, e 4 vezes ao dia) durante 65 dias.

Ciência Rural, v.35, n.1, jan-fev, 2005. 


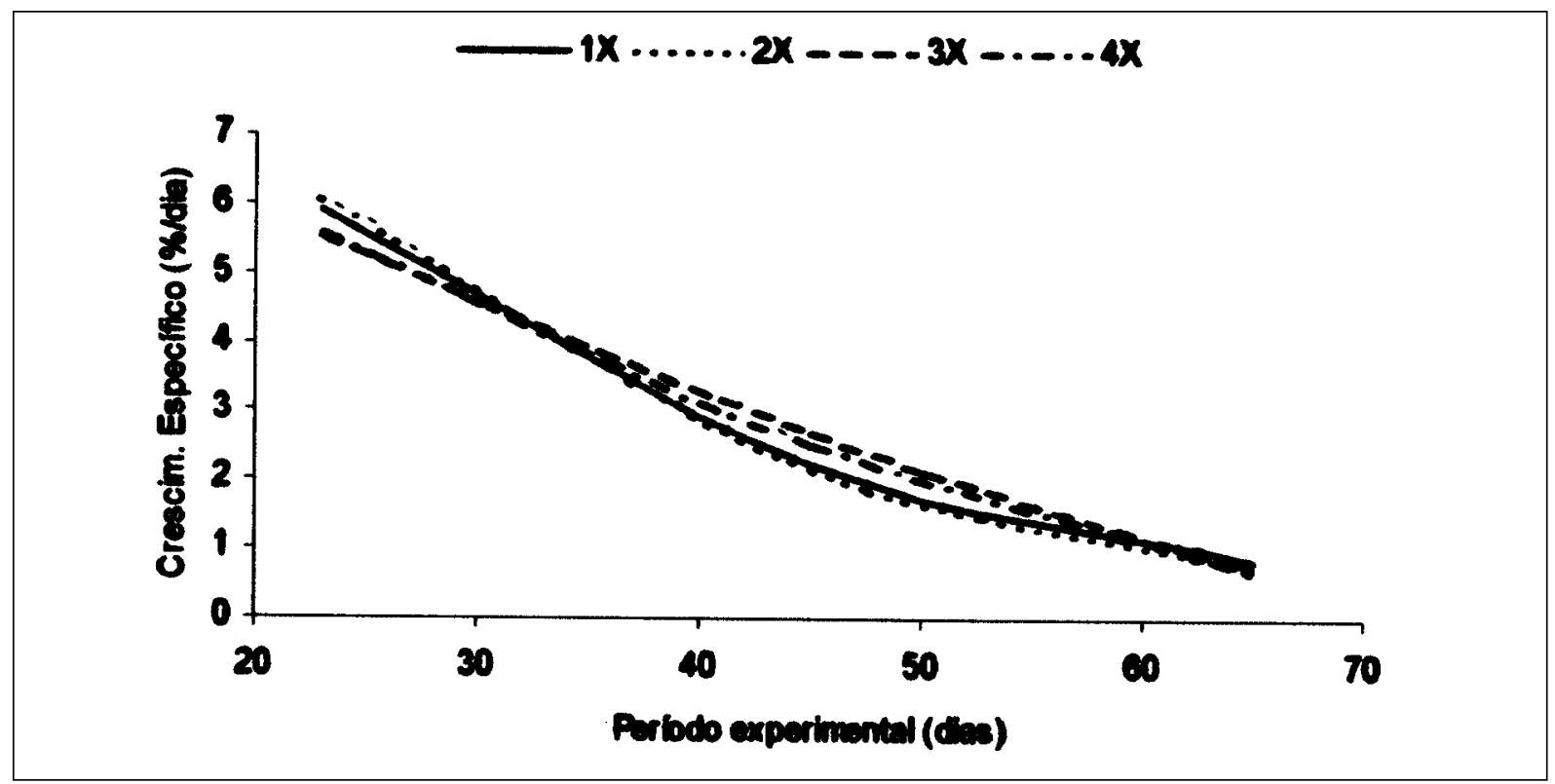

Figura 3 - Crescimento específico de alevinos de jundiá submetidos a diferentes regimes de fornecimento de ração (1, 2, 3, e 4 vezes ao dia) durante 65 dias.

demonstrou o efeito da freqüência alimentar (1, 2, 3 e 4 vezes ao dia) em "sunfish” híbrido (fêmeas Lepomis cyanellus X machos $\mathbf{L}$. macrochirus), com resultados satisfatório para as freqüências 3 e 4 vezes ao dia (WANG et al., 1998). Por outro lado LEE et al. (2000), trabalhando com "rockfish”, Sebastes schlegeli, obtiveram maior ganho de peso em peixes alimentados apenas uma vez ao dia, registrando ainda maior acúmulo de lipídios corporais nesses peixes.

APPEL (2000), trabalhando com juvenis de jundiá, comparou o fornecimento automático e contínuo de alimento com o fornecimento manual duas vezes ao dia. Os resultados de crescimento obtidos por este autor foram semelhantes aos observados no presente estudo, sugerindo que o jundiá possui capacidade de armazenamento de alimento por período relativamente mais prolongado. Um aspecto relevante na consideração dos resultados obtidos nesse experimento, e que dificilmente pode ter sua importância quantificada, refere-se à maior possibilidade de acompanhamento e observação do comportamento dos peixes com o aumento da freqüência alimentar. Durante as primeiras semanas de vida o crescimento dos peixes é muito acelerado, facilmente dobrando seu peso em poucos dias. A ocorrência de problemas nesta fase da criação, como a instalação de alguma doença ou proliferação de algum parasita, dá-se de forma rápida e a sua detecção com antecipação de horas pode fazer muita diferença no seu controle. Contudo, o fornecimento mais freqüente do alimento permite ao produtor reconhecer e corrigir problemas com maiores chances de sucesso.

\section{CONSIDERAÇÕES FINAIS}

O presente estudo mostra que é possível obter o mesmo crescimento de alevinos de jundiá fornecendo alimento apenas uma vez ao dia quando comparado com duas a quatro vezes. Contudo, o aumento na freqüência de fornecimento do alimento permite maior contato visual do produtor com o peixe, possibilitando melhor acompanhamento do estado de saúde dos animais. Cabe salientar que, nessa última situação, o aumento nos custos referentes à mão de obra também devem ser considerados. Ressalta-se portanto, o alerta para a interpretação dos resultados de trabalhos de pesquisa, não apenas considerando os dados avaliados e apresentados como também todo o contexto que envolve a atividade produtiva.

\section{REFERÊNCIAS BIBLIOGRÁFICAS}

APPEL, HB. Avaliação do crescimento do Jundiá rosa Rhamdia sp, sob dos regimes do arraçoamento. In: SÍMPOSIO BRASILEIRO DE AQÜICULTURA, 11., 2000, Florianópolis, SC. Anais... Florianópolis : Simbraq, 2000. n.p. CD-ROM.

CARNEIRO, P.C.F. et al. Jundiá: um grande peixe para a região Sul. Panorama da Aqüicultura, v.12, p.41-46, 2002.

CARNEIRO, P.C.F. et al. Evaluation of co-feeding live with formulated diet on growth and survival of jundiá larvae, Rhamdia quelen. Scientia Agricola, v. 60, p.615-619, 2003. 
FOLKVORD, A.; OTTERA, H. Effects of initial size distribution, day length, and feeding frequency on growth, survival, and cannibalism in juvelile Atlantic cod (Gadus morhua, L.). Aquaculture, v.114, p.243-260, 1993.

GOMES, L.C. et al. Biologia do jundiá Rhamdia quelen (Teleostei, Pimelodidae). Ciência Rural, v.30, p.170-185, 2000.

LEE, SM. et al. Effects of feeding frequency and dietary moisture content on growth, body composition and gastric evacuation of juvenile Korean rockfish (Sebastes schlegeli). Aquaculture, v.187, p.399-409, 2000.

MURAI, T.; ANDREWS, J.W. Effect of frequency of feeding on growth and food conversion of channel catfish fry. Bulletim of Japanese Society on Science of Fisheries, v.42, p.159-161, 1976.

RUOHONEN, K et al. Effects of feeding frequency on growth and food utilization of rainbow trout (Oncorhynchus mykiss) fed low-fat herring or dry pellets. Aquaculture, v.165, p.111121, 1998.

SILFVERGRIP, A.M.C. A sistematic revision of the neotropical catfish genus Rhamdia (Teleostei, Pimelodidae). 1996. 156f. Thesis (PhD in Zoology) Deparment of Zooloy, Stockholm University and Department of Vertebrate Zoology, Swedish Meseum of Natural History.

THOMASSEN, JM; FJAERA, SO. Studies of feeding frequency for Atlantic salmon (Salmo salar). Aquacultural Engineering, v.15, p.149-157, 1996.

TUCKER, C.S.; ROBINSON, E.H. Feeds and feeding practices. Channel catfish farming handbook. New York : AVI Book, 1991. Cap.10, p.292-315.

WANG, N. et al. Effect of feeding frequency on food consumption, growth, size variation, and feeding pattern of age-O hybrid sunfish. Aquaculture, v.165, p.261-267, 1998 